\title{
Nyquist Stability Analysis of an AC-Grid Connected VSC-HVDC System Using a Distributed Parameter DC Cable Model
}

\author{
Yujiao Song and Claes Breitholtz
}

\begin{abstract}
In this paper, a two-terminal VSC-HVDC system embedded in a weak grid ac environment is considered, emphasizing modeling, controller design, and small-signal stability analysis. Traditionally, the de cable is modeled by $\Pi$-sections, implying that care has to be taken when using the model for higher frequencies or in cases of higher cable impedance density, such as submarine cables. Here, a distributed parameter cable model is used to overcome this problem. The VSC-HVDC system can be described as two cascaded blocks. The first block is a transfer function that will differ depending on what input and output variables are considered, but which is in all realistic cases stable. The second block is a feedback loop, where the forward path is a rational function and the return path is a dissipative infinite dimensional function, remaining the same in all cases. The stability is then analyzed, using the Nyquist criterion, in a straightforward manner. Numerical examples are given by the use of MATLAB. The result is that if the VSC-HVDC system using a single $\Pi$-section cable model is stable, so is the VSC-HVDC system using a distributed parameter cable model.
\end{abstract}

Index Terms-Distributed parameter cable model, Nyquist stability criterion, VSC-HVDC system, weak ac environment.

\section{INTRODUCTION}

$\mathbf{V}$ OLTAGE source converter based high voltage direct current transmission (VSC-HVDC) systems have now been in operation since 1997 being a reliable and flexible method of power transmission [1]. A typical application is the integration of renewable energy resources over large geographical areas, such as wind farms [2]. Various aspects of these VSC-HVDC systems have been addressed, especially focusing on control strategy and small-signal stability analysis. In some of them, focus has been on short-term operation of the HVDC systems, emphasizing dynamics and control.

From a system theory point of view, the VSCs involve several linear subsystems, e.g., PI-controllers and linear circuit elements, but also nonlinear subsystems, due to the relations between power and voltage (or current). If large changes in voltage

\footnotetext{
Manuscript received December 31, 2014; revised March 28, 2015; accepted October 12, 2015. Date of publication December 04, 2015; date of current version March 22, 2016. This work was supported by the Chalmers Energy Initiative (CEI). Paper no. TPWRD-01609-2014.

The authors are with the Department of Signals and Systems, Division of Automatic Control, Automation and Mechatronics, Group of Automatic Control, Chalmers University of Technology, Gothenburg SE-412 96, Sweden (e-mail: yujiao@chalmers.se; claesbr@chalmers.se).

Color versions of one or more of the figures in this paper are available online at http://ieeexplore.ieee.org.

Digital Object Identifier 10.1109/TPWRD.2015.2501459
}

levels should be taken into account, e.g., due to severe system faults, nonlinear dynamic models must be used in analysis as well as simulation. A major interest has been in the dynamics close to steady state (implying sinusoidal steady state at the AC-side and constant steady state at the DC-side). Consequently linearized dynamic models have been in focus [3].

However, little attention has so far been paid to evaluating the influence of DC cable dynamics of a VSC-HVDC system, especially when embedded within a weak AC-environment. Typically, DC cables have been modeled by a single $\Pi$-section; thus, the stability of linearized multi-terminal or two-terminal VSC-HVDC systems could be analyzed by straightforward calculation of the system eigenvalues [4], [5]. This is often sufficient, at least when considering the dynamics of short cables and low frequencies. If more general results are required, such as fast system excitation caused by abrupt disturbances and perhaps also in submarine cables (due to increased impedance density, i.e., inductance, capacitance and resistance per unit length), it would be more appropriate to use a distributed parameter cable model together with transfer functions (or finite order state space) linearized VSC models.

In this article, the VSC-HVDC system embedded in a weak-AC environment is modeled, comprising VSCs and AC-side dynamics, together with a distributed parameter DC cable model, based on the one-dimensional damped wave equation. The system is linearized around an arbitrary operational point and the resulting linear infinite-dimensional model is based on the use of transfer functions. The VSC-HVDC system can be described as two cascaded systems. One is a forward combination of transfer functions that will be depending on what input signal and what output signal are considered, which in cases of reasonably large AC-grid short circuit ratios (SCR) is input to output stable. The second is a feedback system, where the forward subsystem is finite-dimensional and the return subsystem only depends on the infinite-dimensional cable model. The stability of this feedback system, which remains the same for all combinations of input and output signals, is analyzed by use of the Nyquist criterion. Schemes and equations are deduced and simulation results in frequency domain will be shown as well. The purpose is to provide a tool for small-signal analysis of the total VSC-HVDC system, including a cable model that is valid independently of cable length and impedance density or what frequencies can be considered due to the cable model approximation. 


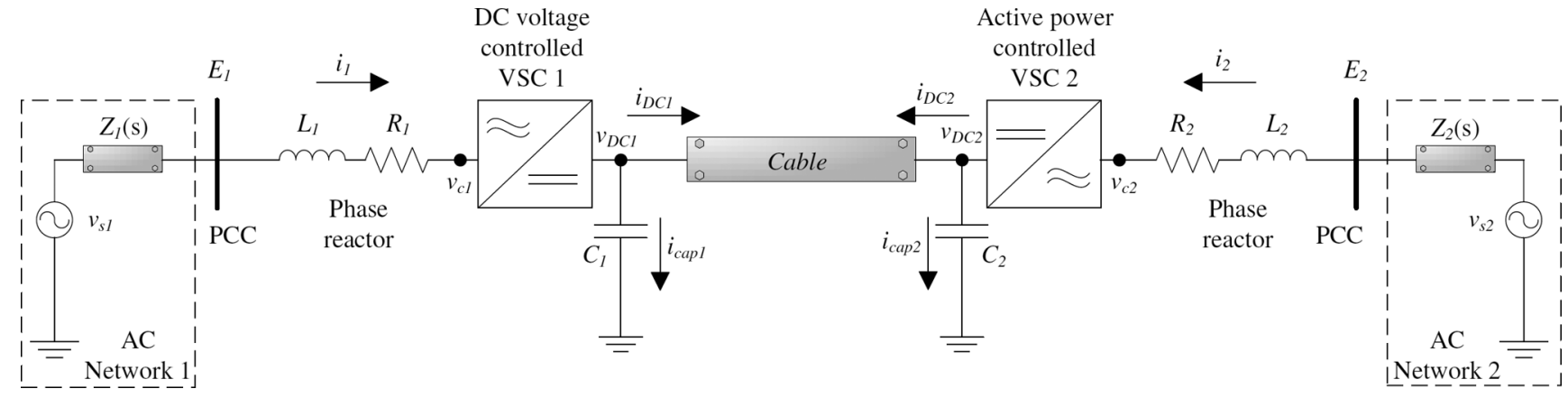

Fig. 1. Standard HVDC-VSC system embedded in a weak AC-environment. $v_{s}$ is the source voltage; $E$ is the PCC voltage; $i$ is the converter input current; $v_{c}$ is the converter voltage; $E_{c}$ is the converter measured PCC voltage; $i_{c}$ is the converter measured input current; $Z(s)$ is the AC-grid impedance.

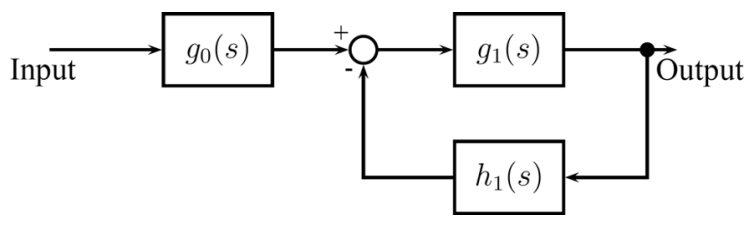

Fig. 2. Block diagram: $g_{0}$ is the forward transfer function, $g_{1} h_{1}$ is the open loop function of the feedback loop $g_{1} /\left(1+g_{1} h_{1}\right)$.

\section{PRELIMINARIES}

The system under study is depicted in Fig. 1, where the weak AC-grids are modeled by the impedance matrices $Z_{1}(\mathrm{~s})$ and $Z_{2}(\mathrm{~s})$ at the synchronous frame, respectively. The so-called synchronous frame is oriented to be in alignment with the voltage direction, due to the Phase Locked Loops (PLL) [6]. In this paper, the PLL used for such synchronization is considered. At the AC-side of the VSCs, the series inductances $\left(L_{1}, L_{2}\right)$ and resistances $\left(R_{1}, R_{2}\right)$ represent the $\mathrm{AC}$ reactors and the power losses in the converters. The DC cable is interpreted as a distributed parameter model. The shunt capacitors at the terminals are denoted $C_{1}, C_{2}$, respectively.

In standard VSC-HVDC systems, one converter station (VSC1) is assigned the duty of DC-voltage controller to secure the stability of the DC-Bus voltage; the other station (VSC2) serves as the active power controller to guarantee and balance the power exchanges [7]. Further, in each VSC, it is possible to control the grid voltage at the AC-side, which in this paper is done by a P-controller.

If the DC cable is modeled by a single $\Pi$-section, the stability of the system can be investigated by an eigenvalue calculation. However, the high-frequency information is not necessarily well interpreted by the single $\Pi$-section cable model and thus poorly suited to analyze the real dynamic influence of the DC cable. The new standard VSC-HVDC model includes the distributed parameter cable model, formulating the transfer function into the block diagram as shown in Fig. 2. Therefore, if $g_{1}(s)$ is a rational function of 's' and the infinite-dimensional transfer function $h_{1}(s)$ represents a dissipative system, the feedback loop stability could be analyzed using the Nyquist criterion.

The VSC-model is derived in the so-called $d q$ reference plane as well. The two VSCs are assumed to be ideal and symmetrical, having a switching frequency of $1[\mathrm{kHz}]$. Henceforth, the units for voltage, current and active power are $[\mathrm{kV}],[\mathrm{kA}]$ and $[\mathrm{MW}]$, respectively, and all reference signals $x$ are expressed as $x^{r e f}$; the differential operator with respect to time is expressed by ' $p$ ', and the Laplace form of a time domain variable $x(t)$ is denoted $\tilde{x}(s)$.

\section{SYSTEM MODEL}

In this section, the dynamic equations of a VSC is given in the power invariant $d q$-reference frame, where the frame is chosen to be aligned with the voltage direction, i.e., $E_{c q 10}=$ $E_{c q 20}=0 . E_{c q 10}$ and $E_{c q 20}$ are the steady state of the converter measured point of common coupling (PCC) voltage. The AC-grid frequency is assumed to be constant, i.e., $\omega_{0}=2 \pi f_{0}$ and $f_{0}=50[\mathrm{~Hz}]$. Since the VSCs are assumed to be symmetric, the variables and parameters in this section would not be subscribed by the numbers 1 and 2, used to denote the different VSCs.

The local controller of the VSC is separated into two cascaded parts: inner current controller and outer controller. The inner current controller provides the voltage reference $\left(v_{c d}^{r e f}\right.$ and $v_{c q}^{r e f}$ ) to the pulse width modulator (PWM). The $d$-axis outer controller is used to track the reference of either DC voltage or active power, generating the $d$-axis current reference $\left(i_{d}^{r e f}\right)$ to the inner current loop. The $q$-axis outer controller is used to limit the voltage drop of the PCC voltage by a P-controller [8], generating the $q$-axis current reference $\left(i_{q}^{r e f}\right)$ to the inner current loop as well.

\section{A. Inner Current Loop}

The AC-current dynamics in the $d q$ frame is given as follows, where $i_{c d}, i_{c q}$ are the measured converter input currents; $E_{c d}$, $E_{c q}$ are the measured PCC voltages; $v_{c d}, v_{c q}$ are the measured converter voltages:

$$
\begin{aligned}
& L \cdot \frac{\mathrm{d} i_{c d}}{\mathrm{~d} t}=-R \cdot i_{c d}+\omega_{0} L \cdot i_{c q}+E_{c d}-v_{c d} \\
& L \cdot \frac{\mathrm{d} i_{c q}}{\mathrm{~d} t}=-R \cdot i_{c q}-\omega_{0} L \cdot i_{c d}+E_{c q}-v_{c q} .
\end{aligned}
$$

The current controller consists of one PI-controller and two feed forward signals i.e., the PCC voltage and the cross-coupling current [9]. The reference voltages $\left(v_{c d}^{r e f}, v_{c q}^{r e f}\right)$ are given by:

$$
\begin{aligned}
& v_{c d}^{r e f}=-\left(K_{p}+\frac{K_{i}}{p}\right)\left(i_{d}^{r e f}-i_{c d}\right)+E_{c d}+\omega_{0} L \cdot i_{c q} \\
& v_{c q}^{r e f}=-\left(K_{p}+\frac{K_{i}}{p}\right)\left(i_{q}^{r e f}-i_{c q}\right)+E_{c q}-\omega_{0} L \cdot i_{c d}
\end{aligned}
$$


Due to the switching action of PWM inside the converter, a delay of half a switching period appears, where $T_{s w}=1 /\left(2 f_{s w}\right)=0.5[\mathrm{~ms}]$. After having designed the inner current loop to be relatively slow, the delay may be ignored during the analysis of system dynamics, i.e., $v_{c d}^{r e f} \approx v_{c d}$ and $v_{c q}^{r e f} \approx v_{c q}$ [8]. Consequently, the dynamics between the $d$-and $q$-axes is decoupled at the inner current loop.

The PI-parameters are designed as $K_{p}=\alpha_{c} L$ and $K_{i}=$ $\alpha_{c} R$, where $\alpha_{c}$ is the bandwidth of the inner current loop and the phase reactor pole of $-R / L$ is thus canceled by the inner current PI-controller. Note that the unavoidable uncertainties in the AC-side model parameters, $\mathrm{L}$ and $\mathrm{R}$, from a practical point of view are assumed to be quite small. Hence, these uncertainties will have only a minor impact on the inner current loop performance. In addition, since the outer loop bandwidth is usually designed to be at least ten times smaller than $\alpha_{c}$, the effect caused by the inner loop parameter uncertainty does not influence the entire system dynamics very much. Therefore, the design of the inner current loop PI-controller based on model parameters is well justified. Consequently, the linearized inner current closed loop in the Laplace domain is:

$$
\begin{aligned}
\Delta \tilde{i}_{c d}(s) & =\frac{\alpha_{c}}{s+\alpha_{c}} \Delta \tilde{i}_{d}^{r e f}(s) \\
\Delta \tilde{i}_{c q}(s) & =\frac{\alpha_{c}}{s+\alpha_{c}} \Delta \tilde{i}_{q}^{r e f}(s) .
\end{aligned}
$$

\section{B. Direct Voltage Control Loop}

The DC voltage is determined by the capacitor charging power that is the difference between the input active power to the VSC (assuming that the VSC is power lossless) and the DC load power, which gives:

$$
\begin{aligned}
\frac{\mathrm{d}}{\mathrm{d} t}\left(\frac{1}{2} C \cdot v_{D C}^{2}\right) & =P-P_{\text {load }} \\
\Rightarrow \quad C \cdot v_{D C 0} \cdot \frac{\mathrm{d} \Delta v_{D C}}{\mathrm{~d} t} & =\Delta P-\Delta P_{\text {load }} .
\end{aligned}
$$

Therefore, the linearized expressions of the input active power and the DC load power are required, where the conjugate value of $x$ is denoted by $x^{*}$ :

$$
\begin{aligned}
P & =\operatorname{Re}\left\{\left(E_{0}+\Delta E_{c d}+j \Delta E_{c q}\right)\left(i_{d 0}+\Delta i_{c d}+j\left(i_{q 0}+\Delta i_{c q}\right)\right)^{*}\right\} \\
& \approx \underbrace{E_{0} i_{d 0}}_{P_{0}}+\underbrace{E_{0} \Delta i_{c d}+i_{d 0} \Delta E_{c d}+i_{q 0} \Delta E_{c q}}_{\Delta P} \\
P_{\text {load }} & =\left(v_{D C 0}+\Delta v_{D C}\right)\left(i_{D C 0}+\Delta i_{D C}\right) \\
& \approx \underbrace{v_{D C 0} i_{D C 0}}_{P_{\text {load } 0}}+\underbrace{v_{D C 0} \Delta i_{D C}+i_{D C 0} \Delta v_{D C}}_{\Delta P_{\text {load }}} .
\end{aligned}
$$

Equation (9) shows that $\Delta P$ is proportional to $\Delta i_{c d}$ and independent of $\Delta i_{c q}$. Therefore, the output of the DC voltage controller, i.e., the d-axis reference current, could be designed through the reference input active power. The reference input active power is the sum of a PI-controller operating on the error of the DC voltage square and the filtered feed forward DC load power (with bandwidth $\alpha_{f}$ ):

$$
P^{r e f}=\left(K_{p d}+\frac{K_{i d}}{p}\right) \cdot \nu+\frac{\alpha_{f}}{p+\alpha_{f}} P_{l o a d}
$$

$$
\begin{aligned}
\nu & =\frac{\left(v_{D C}^{r e f}\right)^{2}-v_{D C}^{2}}{2} \text { and } i_{d}^{r e f}=\frac{P^{r e f}}{E_{c d}} \\
\Delta \tilde{P}^{r e f} & =v_{D C 0} F_{d}\left(\Delta \tilde{v}_{D C}^{r e f}-\Delta \tilde{v}_{D C}\right)+F_{f} \Delta \tilde{P}_{l o a d} \\
F_{d} & =K_{p d}+\frac{K_{i d}}{s} \text { and } F_{f}=\frac{\alpha_{f}}{s+\alpha_{f}} \\
\Delta \tilde{i}_{d}^{r e f} & =\frac{1}{E_{0}} \Delta \tilde{P}^{r e f}-\frac{P_{0}}{E_{0}^{2}} \Delta \tilde{E}_{c d} .
\end{aligned}
$$

Ignoring both the low-pass filter (LPF) in (13) and the inner current loop dynamics i.e., $\Delta i_{c d}=\Delta i_{d}^{r e f}$, designing the PI-controller parameters as $K_{p d}=2 \hat{C} \zeta \omega_{n d}$ and $K_{i d}=\hat{C} \omega_{n d}^{2}$, the transfer function from $\Delta \tilde{v}_{D C}^{r e f}$ to $\Delta \tilde{v}_{D C}$ is:

$$
\frac{\Delta \tilde{v}_{D C}}{\Delta \tilde{v}_{D C}^{r e f}} \approx \frac{2 \zeta \omega_{n d} \frac{\hat{C}}{C} s+\omega_{n d}^{2} \frac{\hat{C}}{C}}{s^{2}+2 \zeta \omega_{n d} \frac{\hat{C}}{C} s+\omega_{n d}^{2} \frac{\hat{C}}{C}} .
$$

The approximation is based on the fact that the inner current loop dynamics is designed to be ten times faster than the DC voltage loop, also assuming that the DC load power signal has lower magnitude in the higher frequency band.

$\hat{C}$ is the estimated DC-shunt capacitance and $C$ is the actual physical shunt capacitance. The damping ratio $\zeta$ and natural frequency $\omega_{n d}$ are amplified by $\sqrt{\hat{C} / C}$, compared to the designed parameters. Since the physical shunt capacitance $C$ contains the DC cable shunt capacitance, which is assumed to be larger than $\hat{C}$, then $\zeta$ and $\omega_{n d}$ should be higher than the designed value in order to compensate for the error. In this paper, the influence of such parameter uncertainty of $C$ is not considered. $\zeta$ and $\omega_{n d}$ are chosen to be 1 and $0.4 \alpha_{d}$, which guarantees that the DC voltage loop bandwidth of (15) is $\alpha_{d}$ [10].

\section{Active Power Control Loop}

For the outer controller of transmitted active power, again a PI-controller is used. The controller parameters are designed by inner current loop pole cancelation i.e., $K_{p p}=\alpha_{p} / \alpha_{c}, K_{i p}=$ $\alpha_{p}$. The bandwidth of the outer loop is chosen as $\alpha_{p}=0.2 \alpha_{d}$. Therefore, the DC-voltage does not display large oscillations during the variations of transmitted active power. The linearized differential equations of the active power control loop are derived as follows:

$$
\begin{aligned}
i_{d}^{r e f} & =\left(K_{p p}+\frac{K_{i p}}{p}\right) \frac{\left(P^{r e f}-P\right)}{E_{c d}} \\
\Rightarrow \Delta \tilde{i}_{d}^{r e f} & =\frac{1}{E_{0}}\left(K_{p p}+\frac{K_{i p}}{s}\right)\left(\Delta \tilde{P}^{r e f}-\Delta \tilde{P}\right) .
\end{aligned}
$$

\section{Alternating Voltage Control Loop}

The idea of the AC-voltage controller is to compensate for the AC-voltage drop, i.e., to increase the reactive power at PCC while the AC voltage is lower than the reference $\mathrm{PCC}$ voltage [11]. A P-controller is applied and thus the q-axis reference current is:

$$
\Delta \tilde{i}_{q}^{r e f}=K_{p a} \cdot\left(\Delta \tilde{E}^{r e f}-\Delta \tilde{E}_{c d}\right) .
$$

It is also possible to use a PI-controller instead of a P-controller. The reason why this is not done is that there is no accuracy specification for the $\mathrm{AC}$ voltage control and that the addition of an extra integral control action will not promote system 


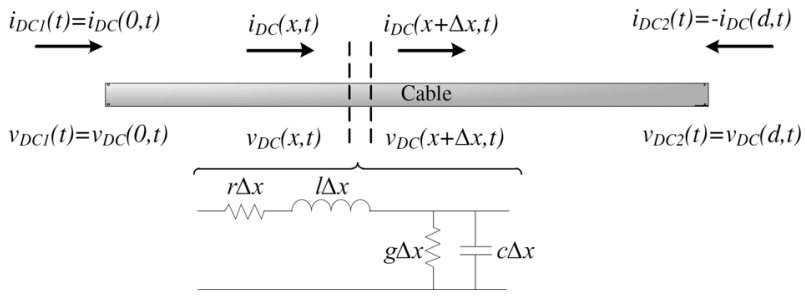

Fig. 3. DC cable model: since the current from the AC to the DC side is defined as positive, $i_{D C 1}=i_{D C}(0, t)$ and $i_{D C 2}=-i_{D C}(d, t)$.

stability. Therefore, in this paper, a P-controller is applied to control the AC voltage.

\section{E. AC-Grid and PLL}

A PLL is applied to track the rotating phase angle $\theta^{\text {ref }}$, which is used to transform the converter $d q$-frame from the stationary $\alpha \beta$-frame. At steady state, the $q$-part of the PCC voltage at the converter $d q$-frame is zero. Due to the assumption of constant $\mathrm{AC}$-grid frequency, a $\mathrm{P}$-controller is thus sufficient to track the reference $\mathrm{AC}$-grid phase. The transfer function from $\Delta E_{q}$ to $\Delta \theta$, relationships between voltages $\left(\Delta E_{d}, \Delta E_{q}\right)$, currents $\left(\Delta i_{d}, \Delta i_{q}\right)$ of the grid $d q$-frame, voltages $\left(\Delta E_{c d}, \Delta E_{c q}\right)$, and currents $\left(\Delta i_{c d}, \Delta i_{c q}\right)$ of the converter $d q$-frame are given by [8]:

$$
\begin{aligned}
\Delta \tilde{\theta} & =\frac{\alpha_{p l l}}{s+\alpha_{p l l}} \Delta \tilde{E}_{q} \\
\Delta \tilde{E}_{c d} & =\Delta \tilde{E}_{d}, \text { and } \Delta \tilde{E}_{c q}=\frac{s}{s+\alpha_{p l l}} \Delta \tilde{E}_{q} \\
\Delta \tilde{i}_{d} & =\Delta \tilde{i}_{c d}+\frac{Q_{0}}{E_{0}^{2}} \frac{\alpha_{p l l}}{s+\alpha_{p l l}} \Delta \tilde{E}_{q} \\
\Delta \tilde{i}_{q} & =\Delta \tilde{i}_{c q}+\frac{P_{0}}{E_{0}^{2}} \frac{\alpha_{p l l}}{s+\alpha_{p l l}} \Delta \tilde{E}_{q} .
\end{aligned}
$$

For a strong AC-grid, the PCC voltage is independent of the current variation, while $\Delta E_{d}$ and $\Delta E_{q}$ are input disturbances of the VSC system. However, for a weak AC-grid, the PCC voltage is related to the current or power variation. In this case, the $d$-axis and $q$-axis dynamics are not independent of each other but correlated through the AC-grid impedance. The AC-grid is modeled by a series $R_{g} L_{g}$-circuit in the synchronous $d q$-frame. The AC-grid dynamics are:

$$
\begin{aligned}
& \Delta \tilde{v}_{s d}-\Delta \tilde{E}_{d}=\left(L_{g} s+R_{g}\right) \Delta \tilde{i}_{d}-\omega_{0} L_{g} \Delta \tilde{i}_{q} \\
& \Delta \tilde{v}_{s q}-\Delta \tilde{E}_{q}=\omega_{0} L_{g} \Delta \tilde{i}_{d}+\left(L_{g} s+R_{g}\right) \Delta \tilde{i}_{q}
\end{aligned}
$$

\section{F. DC Cable}

The conventional method of approximating a transmission line is to replace the line by small cascaded lumped RLGC-sections, as shown in Fig. 3. The terminal voltages and currents are represented by $v_{D C 1}, v_{D C 2}, i_{D C 1}$ and $i_{D C 2}$, respectively. The current from the $\mathrm{AC}$ to the $\mathrm{DC}$ side is defined as positive. The cable parameters are given by $\mathrm{r}, 1, \mathrm{~g}, \mathrm{c}$ and $\mathrm{d}$, which are the cable density of resistance, inductance, conductance, capacitance and cable length.
Define the complex damping factor per unit length $\gamma$ and the wave admittance $Y_{0}$ as:

$$
\begin{aligned}
\gamma(s) & =\sqrt{(c \cdot s+g)(l \cdot s+r)}, \Gamma(s)=d \cdot \gamma(s) \\
Y_{0}(s) & =\sqrt{\frac{c \cdot s+g}{l \cdot s+r}} .
\end{aligned}
$$

By solving the readily derived linear partial differential equations, obtained from Fig. 3. after letting the element $\Delta x \rightarrow 0$ and taking Laplace transforms with respect to time, the relationship between the terminal currents and voltages is formulated [10]:

$$
\begin{aligned}
{\left[\begin{array}{c}
\tilde{i}_{D C 1}(s) \\
\tilde{i}_{D C 2}(s)
\end{array}\right] } & =\left[\begin{array}{cc}
h_{1}(s) & -h_{2}(s) \\
-h_{2}(s) & h_{1}(s)
\end{array}\right] \cdot\left[\begin{array}{c}
\tilde{v}_{D C 1}(s) \\
\tilde{v}_{D C 2}(s)
\end{array}\right] \\
\text { where, } h_{1}(s) & =Y_{0}(s) \operatorname{coth}(\Gamma(s)) \\
h_{2}(s) & =\frac{Y_{0}(s)}{\sinh (\Gamma(s))} .
\end{aligned}
$$

As the cable system itself is dissipative, the functions $h_{1}(s)$ and $h_{2}(s)$ have infinite numbers of stable poles. In addition, the square difference between $h_{1}(s)$ and $h_{2}(s)$ is identically a rational function, i.e., $h_{1}^{2}(s)-h_{2}^{2}(s)=Y_{0}^{2}(s)$.

\section{Block Diagram}

In this section, the two-terminal VSC-HVDC system embedded in strong or weak AC-environments is considered and transformed into the block diagram at Fig. 2.

\section{A. Weak AC-Grid Environment}

In a situation where both VSCs are embedded in the weak AC-grid environment, for each VSC system there are five input signals, i.e., $\Delta v_{s d}, \Delta v_{s q}, \Delta i_{D C}$ and reference signals $\Delta v_{D C 1}^{r e f}$ or $\Delta P_{2}^{r e f}$ and $\Delta E^{r e f}$. Note that $\Delta i_{D C}$ is a state variable in the description of the entire system.

Choose the DC voltage as the VSC system output. Both $\Delta v_{D C}$ and $\Delta i_{D C}$ constitute the interconnection between the VSC and the DC-grid. The preliminary transfer functions from the five inputs to the $\mathrm{DC}$ voltages are given by:

$$
\begin{aligned}
\Delta \tilde{v}_{D C 1}= & g_{11}(s) \Delta \tilde{v}_{D C 1}^{r e f}+g_{12}(s) \Delta \tilde{E}_{1}^{r e f}+g_{13}(s) \Delta \tilde{v}_{s d 1}+ \\
& +g_{14}(s) \Delta \tilde{v}_{s q 1}+g_{15}(s) \Delta \tilde{i}_{D C 1} \\
\Delta \tilde{v}_{D C 2}= & g_{21}(s) \Delta \tilde{P}_{2}^{r e f}+g_{22}(s) \Delta \tilde{E}_{2}^{r e f}+g_{23}(s) \Delta \tilde{v}_{s d 2}+ \\
& +g_{24}(s) \Delta \tilde{v}_{s q 2}+g_{25}(s) \Delta \tilde{i}_{D C 2} .
\end{aligned}
$$

Combine the relationship between the DC-terminal currents and voltages given at (27), and the two-terminal VSC-HVDC system transfer function matrix is readily obtained from:

$$
\begin{gathered}
\Lambda(s)\left[\begin{array}{c}
\Delta \tilde{v}_{D C 1} \\
\Delta \tilde{v}_{D C 2}
\end{array}\right]=\Phi(s) \cdot u \\
\Lambda(s)=\left[\begin{array}{ccccccc}
1-g_{15} h_{1} & g_{15} h_{2} \\
g_{25} h_{2} & 1-g_{25} h_{1}
\end{array}\right] \\
\Phi(s)=\left[\begin{array}{cccccccc}
g_{11} & g_{12} & 0 & 0 & g_{13} & g_{14} & 0 & 0 \\
0 & 0 & g_{21} & g_{22} & 0 & 0 & g_{23} & g_{24}
\end{array}\right]
\end{gathered}
$$


where,

$$
u^{T}=\left[\Delta \tilde{v}_{d c 1}^{r e f} \Delta \tilde{E}_{1}^{r e f} \Delta \tilde{P}_{2}^{r e f} \Delta \tilde{E}_{2}^{r e f} \Delta \tilde{v}_{s d 1} \Delta \tilde{v}_{s q 1} \Delta \tilde{v}_{s d 2} \Delta \tilde{v}_{s q 2}\right]
$$

The MIMO transfer function from inputs to outputs is given as follows, where $\operatorname{adj}(\Lambda)$ denotes the adjoint matrix of $\Lambda$ and $G(s)=\operatorname{adj}(\Lambda) \Phi:$

$$
\begin{aligned}
{\left[\begin{array}{c}
\Delta \tilde{v}_{D C 1} \\
\Delta \tilde{v}_{D C 2}
\end{array}\right] } & =\Lambda^{-1} \Phi \cdot u=\frac{1}{\operatorname{det} \Lambda} \operatorname{adj}(\Lambda) \Phi \cdot u \\
& =\underbrace{\frac{-\frac{g_{15}+g_{25}}{1+Y_{0}^{2} g_{15} g_{25}}}{1-\frac{g_{15}+g_{25}}{1+Y_{0}^{2} g_{15} g_{25}} \cdot h_{1}}}_{\text {Feedback loop } g_{1} / 1+g_{1} h_{1}} \underbrace{\frac{-1}{g_{15}+g_{25}} G(s)}_{\text {Forward function } g_{0}} \cdot u .
\end{aligned}
$$

\section{B. Strong AC-Grid Environment}

While the connected AC-grids are well balanced and strong i.e., the nominal AC-grid voltage is at most subject to small variation, the dynamics of the PLL is ignored. In addition, since the $q$-axis current has no impact on the dynamics at the DC-side (after $d$ - and $q$-dynamics decoupling at the inner current loop), the $q$-axis current reference $\left(\Delta i_{q}^{r e f}\right)$ is thus assumed to be zero.

In this case, for each VSC, there are three input signals i.e., $\Delta v_{s d}, \Delta i_{D C}$ and reference signal $\Delta v_{D C 1}^{r e f}$ or $\Delta P_{2}^{r e f}$. As before, choose the DC voltage as the VSC output. The transfer functions from inputs $\hat{u}=\left[\begin{array}{llll}\Delta \tilde{v}_{D C 1}^{r e f} \Delta \tilde{P}_{2}^{r e f} & \Delta \tilde{v}_{s d 1} \Delta \tilde{v}_{s d 2}\end{array}\right]^{T}$ to the DC voltages are:

$$
\begin{aligned}
& \Delta \tilde{v}_{D C 1}=\hat{g}_{11}(s) \Delta \tilde{v}_{D C 1}^{r e f}+\hat{g}_{13}(s) \Delta \tilde{v}_{s d 1}+\hat{g}_{15}(s) \Delta \tilde{i}_{D C 1} \\
& \Delta \tilde{v}_{D C 2}=\hat{g}_{21}(s) \Delta \tilde{P}_{2}^{r e f}+\hat{g}_{23}(s) \Delta \tilde{v}_{s d 2}+\hat{g}_{25}(s) \Delta \tilde{i}_{D C 2} \\
& {\left[\begin{array}{c}
\Delta \tilde{v}_{D C 1} \\
\Delta \tilde{v}_{D C 2}
\end{array}\right]=\underbrace{\frac{-\frac{\hat{g}_{15}+\hat{g}_{25}}{1+Y_{0}^{2} \hat{g}_{15} \hat{g}_{25}}}{1-\frac{\hat{g}_{15}+\hat{g}_{25}}{1+Y_{0}^{2} \hat{g}_{15} \hat{g}_{25}} \cdot h_{1}}}_{\text {Feedback loop } \hat{g}_{1} / 1+\hat{g}_{1} \hat{h}_{1}} \underbrace{\frac{-1}{\hat{g}_{15}+\hat{g}_{25}} \hat{G}(s)}_{\text {Forward function } \hat{g}_{0}} \cdot \hat{u}}
\end{aligned}
$$

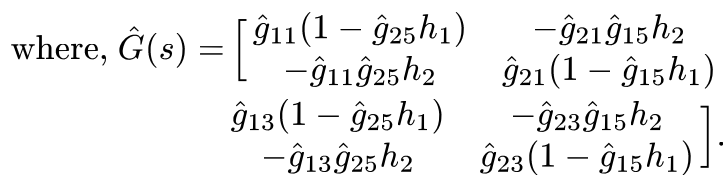

For both weak and strong AC-environment, the transfer functions of a two-terminal VSC-HVDC system, using a distributed parameter DC cable model, can be written in the form $g_{0}$. $g_{1} /\left(1+g_{1} h_{1}\right)$. The forward function $g_{0}$ is determined by the choice of input and output, while the feedback loop is invariant regardless of choice. Using this approach, the small-signal stability of the infinite-dimensional system could be analyzed by the Nyquist criterion.

\section{Stability AnAlysis}

In order to guarantee the stability of the entire system, both the feedback loop and the forward transfer function should be stable. Even though the forward transfer function $g_{0}$ is determined by an input-output combination, it will always be a linear non-feedback combination of rational functions and of $h_{1}$ or $h_{2}$. Thus, the stability of $g_{0}$ is given according to the sign of the real parts of a limited number of poles. The stability of the feedback loop is analyzed by using the Nyquist stability criterion.
Assuming that the closed path $\mathcal{C}$, enclosing all of the right half complex s-plane, has the clockwise positive direction, we can apply the Nyquist criterion: the number of anti-clockwise encirclements around the point -1 in the $g_{1}(s) h_{1}(s)$-plane should, for a stable closed loop system, equal the number of open loop unstable poles of $g_{1}(s)$, as $h_{1}(s)$ is already an input-output stable function [12].

\section{A. Weak AC-Grid Environment}

In this paper, the weak AC-grid is modeled by a series $R_{g} L_{g}$-circuit in the synchronous coordinates and their dynamics are given by (23)-(24).

For the active power controlled VSC, the transfer function $g_{25}$ from $\Delta i_{D C 2}$ to $\Delta v_{D C 2}$ is the same as the VSC connected to a strong AC-grid. Therefore, $g_{25}=\hat{g}_{25}$ and the feedback loop stability is not influenced by the weak AC-grid dynamics at the active power controlled VSC. Similarly, the stability of the active power controlled VSC could be analyzed without considering the DC-side influence. Consequently, the stability analysis of two terminal VSC-HVDC systems embedded in a weak AC-grid can be separated into two parts: the active power controlled VSC and the VSC-HVDC system while the active power controlled VSC is under steady state.

1) Stability of Active Power Controlled VSC: Combining the inner current loop dynamics (5)-(6), active power controlled and AC voltage controlled outer loop dynamics (17)-(18), and the PLL dynamics (20)-(22), then the state-space model of the active power controlled VSC is given by (33)-(34), where the state variables are $x_{a p}^{T}=\left[\Delta i_{c d} \Delta i_{c q} x_{p l l}\right]$, the input variables are $u_{a p}^{T}=\left[\Delta P^{r e f} \Delta E^{r e f} \Delta v_{s d} \Delta v_{s q}\right]$ and the disturbance input variables are $w^{T}=\left[\Delta E_{d} \Delta E_{q}\right]$.

$$
\begin{aligned}
& \dot{x}_{a p}=A_{1} \cdot x_{a p}+B_{u 1} \cdot u_{a p}+B_{w 1} \cdot w \\
& w=C_{w 1} \cdot x_{a p}+D_{w 1} \cdot u_{a p} \\
& A_{1}=\left[\begin{array}{ccc}
-a_{p} & 0 & -a_{p} \cdot \frac{Q_{0}}{E_{0}^{2}} \\
0 & -a_{c} & 0 \\
0 & 0 & -a_{p l l}
\end{array}\right] \\
& B_{u 1}=\left[\begin{array}{cccc}
\frac{\alpha_{p}}{E_{0}} & 0 & 0 & 0 \\
0 & \alpha_{c} K_{p a} & 0 & 0 \\
0 & 0 & 0 & 0
\end{array}\right] \\
& B_{w 1}=\left[\begin{array}{cc}
-a_{p} \cdot \frac{P_{0}}{E_{0}^{2}} & a_{p} \cdot \frac{Q_{0}}{E_{0}^{2}} \\
-a_{c} \cdot K_{p a} & 0 \\
0 & a_{p l l}
\end{array}\right] \text {. }
\end{aligned}
$$

The expressions of $C_{w 1}$ and $D_{w 1}$ are given in the Appendix $\mathrm{A}$. In a strong AC-grid environment, the active power controlled VSC is stable and the system poles are $-\alpha_{c},-\alpha_{p}$ and $-\alpha_{p l l}$.

Since the input signals $u_{a p}$ are independent of the state variable, the linear system stability is not related to the value of $u_{a p}$. Hence, we may assume that $u_{a p}$ is zero. Under weak-AC environment, the block diagram of the active power controlled VSC with zero input signals is depicted in Fig. 4.

According to small gain theorem (SGT) [13], the sufficient condition of stable VSC system is: $\left\|G_{a p}(j \omega)\right\|_{\infty} \cdot\|\Delta A\|_{\infty}<1$, for any $\omega$. By denoting the lowest and highest singular value of any matrix $M$ as $\underline{\sigma}(M)$ and $\bar{\sigma}(M)$, the sufficient condition for 


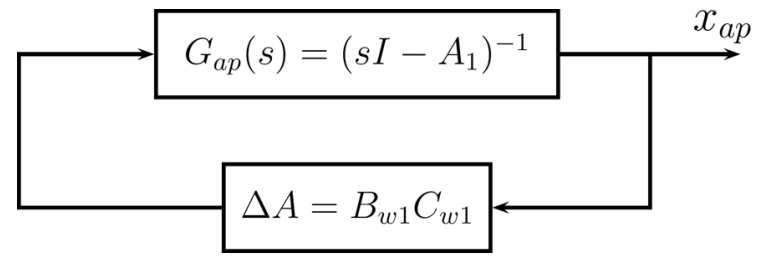

Fig. 4. Block diagram of the active power controlled VSC with zero input signals.

a stable system is rewritten by (35), where $T$ is an invertible scaling matrix and $A_{1}$ has only real eigenvalues:

$$
\begin{aligned}
& \bar{\sigma}\left(\left(j \omega I-A_{1}\right)^{-1}\right) \cdot \bar{\sigma}(\Delta A)<1, \omega \in \mathrm{R}^{+} \\
\Leftrightarrow & \bar{\sigma}(\Delta A)<\frac{1}{\bar{\sigma}\left(\left(j \omega I-A_{1}\right)^{-1}\right)}=\underline{\sigma}\left(j \omega I-A_{1}\right), \omega \in \mathrm{R}^{+} \\
\Leftrightarrow & \bar{\sigma}\left(T^{-1} \cdot \Delta A \cdot T\right)<\underline{\sigma}\left(T^{-1} \cdot A_{1} \cdot T\right) .
\end{aligned}
$$

Since the pole of the state $\Delta i_{c q}$ is at least ten times further away from the imaginary axis compared with the other two states, the dynamics of $\Delta i_{c q}$ is ignored, i.e., $\Delta i_{c q} \approx K_{p a}\left(\Delta E^{r e f}-\Delta E_{c d}\right)$. The state-space model with new state variables $x_{a p n}=\left[\Delta i_{c d} x_{p l l}\right]$ is:

$$
\begin{aligned}
\dot{x}_{a p n} & =\tilde{A}_{1} \cdot x_{a p n}+\tilde{B}_{u 1} \cdot u_{a p}+\tilde{B}_{w 1} \cdot w \\
w & =\tilde{C}_{w 1} \cdot x_{a p n}+\tilde{D}_{w 1} \cdot u_{a p} \\
\tilde{A}_{1} & =\left[\begin{array}{cc}
-a_{p} & -a_{p} \cdot \frac{Q_{0}}{E_{0}^{2}} \\
0 & -a_{p l l}
\end{array}\right] \\
\tilde{B}_{u 1} & =\left[\begin{array}{cc}
\frac{\alpha_{p}}{E_{0}} & 0 \\
0 & 0
\end{array}\right] \quad \tilde{B}_{w 1}=\left[\begin{array}{cc}
-a_{p} \cdot \frac{P_{0}}{E_{0}^{2}} & a_{p} \cdot \frac{Q_{0}}{E_{0}^{2}} \\
0 & a_{p l l}
\end{array}\right] .
\end{aligned}
$$

The expressions of $\tilde{C}_{w 1}$ and $\tilde{D}_{w 1}$ are given in Appendix A. Letting the scaling matrix $T$ be

$$
T=\left[\begin{array}{cc}
0.008 & 0.002 \\
-1 & 1
\end{array}\right]
$$

the minimum singular value of $T^{-1} \cdot A_{1} \cdot T$ and the maximum singular value of $T^{-1} \cdot \Delta A \cdot T$ with respect to $\mathrm{SCR}=1 / L_{g}[\mathrm{p} . \mathrm{u}$.] are given in Fig. 5, where the parameters for the case study are listed in Tables I and II. The P-controller parameter of the AC voltage controller is $K_{p a}=0.1$.

Since $A_{1}$ and $T$ are constant matrices, the minimum singular value of $T^{-1} \cdot A_{1} \cdot T$ is constant and equal to 28.38 . As shown in Fig. 5, for SCR $>2.7$, the maximum singular value of $T^{-1} \cdot \Delta A \cdot T$ is smaller than the minimum singular value of $T^{-1} \cdot A_{1} \cdot T$ and thus the active power controlled VSC is stable. It should be mentioned that the inequality SCR $>2.7$ is a sufficient condition for the stability of the VSC system. For SCR lower than 2.7, however, the system could be stable as well, as can be checked by eigenvalue calculation.

The step response from $\Delta P_{2}^{r e f}$ to $\Delta P_{2}$ in different $\mathrm{AC}$ environments is given in Fig. 6 . For cases with strong AC-grid and for $\mathrm{SCR}=5$, as proved by the SGT, the active power controlled VSC system is stable. However, for SCR $=2$ and $\mathrm{SCR}=1.3$, even though the $\mathrm{SGT}$ is violated, the system is still stable since the SGT is a sufficient but not necessary condition to prove system stability. It shows that, for different SCR, the

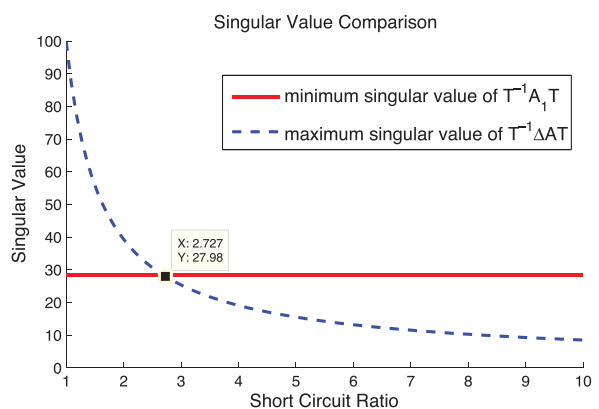

Fig. 5. Singular value with respect to SCR: $\underline{\sigma}\left(T^{-1} A_{1} T\right)$ is a constant and marked by the red curve; $\bar{\sigma}\left(T^{-1} \Delta A T\right)$ decreases with respect to the increased SCR and is marked by the blue curve. The cross point of those two curves is around $\mathrm{SCR}=2.69$ and for $\mathrm{SCR} \geq 2.7, \bar{\sigma}\left(T^{-1} \Delta A T\right)<\underline{\sigma}\left(T^{-1} A_{1} T\right)$ and thus the closed loop system (shown in Fig. 4) is stable.

TABLE I

BANDWIDTH DESIGN OF VSC-HVDC SYSTEM

\begin{tabular}{|l|c|c|c|}
\hline Inner current loop & $\alpha_{c}$ & 4p.u. & $400 \pi[\mathrm{rad} / \mathrm{s}]$ \\
\hline Active power outer loop & $\alpha_{p}$ & 0.1 p.u. & $10 \pi[\mathrm{rad} / \mathrm{s}]$ \\
\hline DC voltage outer loop & $\alpha_{d}$ & $0.4 p . u$. & $40 \pi[\mathrm{rad} / \mathrm{s}]$ \\
\hline PLL loop & $\alpha_{p l l}$ & 0.4 p.u. & $40 \pi[\mathrm{rad} / \mathrm{s}]$ \\
\hline
\end{tabular}

TABLE II

PARAMETER OF VSC-HVDC SYSTEM

\begin{tabular}{|l|c|c|l|}
\hline Cable distance & $\mathrm{d}$ & 50 & $\mathrm{~km}$ \\
\hline Cable inductance density & $\mathrm{l}$ & 9 & $\mathrm{mH} / \mathrm{km}$ \\
\hline Cable capacitance density & $\mathrm{c}$ & 0.207 & $\mu \mathrm{F} / \mathrm{km}$ \\
\hline Cable resistance density & $\mathrm{r}$ & 0.01 & $\Omega / \mathrm{km}$ \\
\hline Cable conductance density & $\mathrm{g}$ & 0 & $\mathrm{~S} / \mathrm{km}$ \\
\hline Phase reactor inductance & $L$ & 53 & $\mathrm{mH}$ \\
\hline Phase reactor resistance & $R$ & 0.167 & $\Omega$ \\
\hline Weak AC-grid inductance & $L_{g}$ & 53.1 & $\mathrm{mH}$ \\
\hline Weak AC-grid resistance & $R_{g}$ & 1.11 & $\Omega$ \\
\hline DC shunt capacitor & $C$ & 33 & $\mu \mathrm{F}$ \\
\hline Rated AC voltage $(d q$-frame) $)$ & $v_{s d 0}$ & 200 & $\mathrm{kV}$ \\
\hline Rated DC voltage & $v_{D C}$ & 300 & $\mathrm{kV}$ \\
\hline Rated transmission power & $P_{0}$ & 600 & $\mathrm{MW}$ \\
\hline System frequency & $f_{0}$ & 50 & $\mathrm{~Hz}$ \\
\hline
\end{tabular}

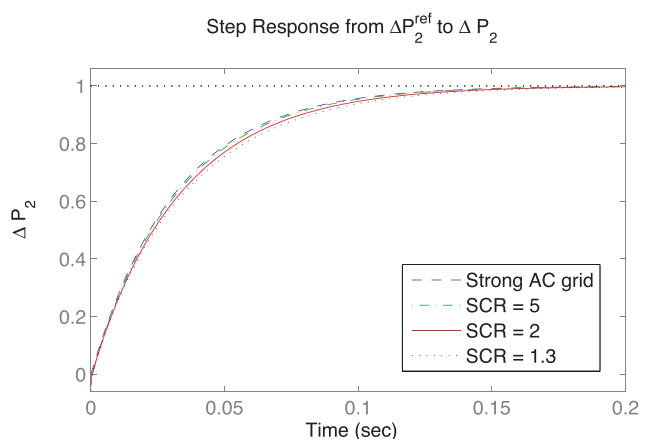

Fig. 6. Step response of the active power controlled VSC with input $\Delta P_{2}^{\text {ref }}$ and output $\Delta P_{2}$ : blue curve is the strong AC-grid case; green curve is the case with $\mathrm{SCR}=5$; red curve is the case with $\mathrm{SCR}=2$; black curve is the case with $\mathrm{SCR}=1.3$.

system is stable but the settling time is longer as the AC-grid is weaker (SCR is lower).

For weak AC-grids with other topology, such as shunt or series $L_{g} C_{g}$-circuits, the SGT could also be applied and yield a 
sufficient condition for the stability analysis. However, the disturbance matrix $\Delta A$ then needs to be reformulated according to the changed AC-grid dynamics.

2) Stability of DC Voltage Controlled VSC: As concluded above, the stability of the active power controlled VSC does not influence the stability of the feedback loop $g_{1} /\left(1+g_{1} h_{1}\right)$ since $g_{25}=\hat{g}_{25}$. In this subsection, assume that the active power controlled VSC is in steady state. On the other side, the DC voltage controlled VSC is connected to a weak AC-grid, which is modeled by a series $L_{g} R_{g}$-circuit.

One ח-Section DC Cable Model: While the DC cable is modeled by a single $\Pi$-section, the VSC-HVDC system without considering the dynamics of active power controlled VSC, could be modeled by an eighth order state space model. The state variables are $x_{d v}^{T}=\left[\Delta i_{c d} \Delta i_{c q} \Delta v_{D C} x_{d v 4} x_{d v 5} x_{p l l} \Delta i_{D C} \Delta v_{D C}^{a p}\right]$, where $x_{d v 4}$ is the integral action of the DC-voltage controller, $x_{d v 5}$ is the dynamics of the LPF of the forward DC-load power, and $\Delta v_{D C}^{a p}$ is the DC voltage at the active power controlled VSC side. The input variables are $u_{d v}^{T}=\left[\Delta v_{D C}^{r e f} \Delta E^{r e f} \Delta v_{s d} \Delta v_{s q}\right]$ and the disturbance input variables are $w^{T}=\left[\Delta E_{d} \Delta E_{q}\right]$. $u_{d v}^{r e f}$ are the first two reference signals of the input vector $u_{d v}$.

Combining the inner current loop dynamics (5)-(6), the DC-voltage dynamics (8), the DC voltage controlled outer loop dynamics (13)-(14), the AC voltage controlled outer loop dynamics (18), the PLL dynamics (20)-(22), the DC-current dynamics due to series $R_{D C} L_{D C}$-circuit and the active power controlled VSC dynamics given by $g_{25}(s)$, then the state-space model of the VSC-HVDC system with one $\Pi$-section DC cable is:

$$
\begin{aligned}
\dot{x}_{d v} & =A_{2} \cdot x_{d v}+B_{u 2} \cdot u_{d v}^{r e f}+B_{w 2} \cdot w \\
w & =C_{w 2} \cdot x_{d v}+D_{w 2} \cdot u_{d v}
\end{aligned}
$$

Expressions of the matrices $A_{2}, B_{u 2}, B_{w 2}, C_{w 2}$ and $D_{w 2}$ are given in Appendix B. $A_{2}, B_{u 2}$ and $B_{w 2}$ do not depend on the SCR value; however, $C_{w 2}$ and $D_{w 2}$ rely on the SCR. The parameters of the DC cable are listed in Table II, where $L_{D C}=l \cdot d$ and $R_{D C}=r \cdot d$. The stability of the VSC-HVDC system with one $\Pi$-section DC cable model can be analyzed by the eigenvalues of $A_{2}+B_{w 2} C_{w 2}$. It is straightforward to calculate that for SCR $<4$, assuming the parameter values from Table II, the VSC-HVDC system would become unstable due to the weak AC-grid impedance but not for cases with higher SCR.

Distributed Parameter DC Cable Model: While the DC cable is modeled by (27), it is hard to use eigenvalue analysis to study the system stability since there are an infinite number of poles. Therefore, the Nyquist criterion is applied to analyze the stability of the system, as shown in the block diagram in Fig. 2.

As claimed in Section IV, the VSC-HVDC system can be described by two cascaded systems: one is a forward combination of transfer functions, which depends on the considered input and output. The second is a feedback loop which is unique to all input-output combinations. For an active power controlled VSC of this case study, the forward functions are stable at least for SCR > 2.7. For a DC voltage controlled VSC, the forward function stability will be analyzed in a case study.
In the case study setup, the VSC-HVDC system parameters and nominal steady states are listed in Table II. However, reset the cable inductance density to be $10.2[\mathrm{mH} / \mathrm{km}]$, which renders the one $\Pi$-section cable model based VSC-HVDC system unstable, and the SCR of the AC-grid is still chosen to be 4 . For the case with the distributed parameter DC cable model, the forward function stability and the function $g_{15}$ are determined by the new state-space model, where $x_{d v n}$ comprises the first six components of $x_{d v}$, the input signal is $\Delta i_{D C}$ and the output signal is $\Delta v_{D C}$ :

$$
\begin{aligned}
\dot{x}_{d v n} & =A_{3} \cdot x_{d v n}+B_{3} \cdot \Delta i_{D C} \\
y & =C_{3} \cdot x_{d v n} \\
g_{15} & =C_{3}\left(s I-A_{3}\right)^{-1} B_{3}
\end{aligned}
$$

where $A_{3}$ is the sub-square matrix of $A_{2}+B_{w 2} C_{w 2}$ with the first 6 columns and first 6 rows and $\mathrm{c}=0[\mu \mathrm{F}]$ (since the cable capacitance should not be included in the VSC dynamics itself), and $B_{3}, C_{3}$ are:

$$
\begin{aligned}
B_{3}^{T} & =\left[\begin{array}{llllll}
0 & 0 & -\frac{1}{C} & 0 & a_{f} v_{D C 0} & 0
\end{array}\right] \\
C_{3} & =\left[\begin{array}{llllll}
0 & 0 & 1 & 0 & 0 & 0
\end{array}\right] .
\end{aligned}
$$

It is straightforward to prove that the above system (40) is minimum order, i.e., the system is both controllable and observable. Therefore, the stability of the forward function $g_{0}=$ $-1 / g_{15}+g_{25} G(s)$ is determined by the zeros of $g_{15}+g_{25}$. The expression of $g_{25}$ is given at (42) due to $g_{25}=\hat{g}_{25}$ and the expression of $g_{15}$ is given by (41). In this case study, the zeros of $g_{15}+g_{25}$ are $-30,-41 \pm 66 j,-119,-1265$, and -466180 ; thus the forward function $g_{0}(s)$ is always stable regardless of the input and output signals.

The feedback loop stability is studied using the Nyquist criterion. The number of unstable poles of $g_{1} h_{1}$ is determined by $g_{1}=-g_{15}+g_{25} / 1+Y_{0}^{2} g_{15} g_{25}$, due to the fact that $h_{1}(s)$ is dissipative, containing an infinite number of stable poles. The poles of $g_{1}$ are: $173,-0.98,-25 \pm 22 j,-119,-434,-1125$ and -449937 . Therefore, there is one unstable pole of the open loop function of $g_{1} /\left(1+g_{1} h_{1}\right)$. In order to guarantee the stability of the closed loop function, the Nyquist plot should, anti-clockwise, encircle the critical point $(-1,0)$ once. For one $\Pi$-section cable model, the equivalent cable function of $h_{1}(s)$ is depicted by $h_{\pi 1}(s)=c s \cdot d / 2+1 /(l s+r) / d$. The Nyquist curve of open loop function $g_{1} h_{1}(j \omega)$ and $g_{1} h_{\pi 1}(j \omega)$ is given by Fig. 7 .

Fig. 7(a) shows that in the low frequency band, both cable models provide similar Nyquist curves. However, in this case study, Fig. 7(b) shows that for the distributed parameter cable model, the Nyquist curve will anti-clockwise encircle the critical point $(-1,0)$, but for the one $\Pi$-section cable model, the Nyquist curve, instead, will clockwise encircle the critical point once. Therefore, the VSC-HVDC system with distributed parameter DC cable model is stable, whereas the VSC-HVDC system with one $\Pi$-section DC cable model has two unstable poles, i.e., $0.7 \pm 1.78 j$, calculated by the eigenvalue of the weak AC-grid connected VSC-HVDC system state matrix $A_{2}+$ $B_{w 2} C_{w 2}$. This is because, for $\omega<1 / d / \sqrt{l c / 2}=\omega_{r f}$ (resonance frequency of one $\Pi$-section cable), $\left|h_{1}(j \omega)\right|>\left|h_{\pi 1}(j \omega)\right|$ 


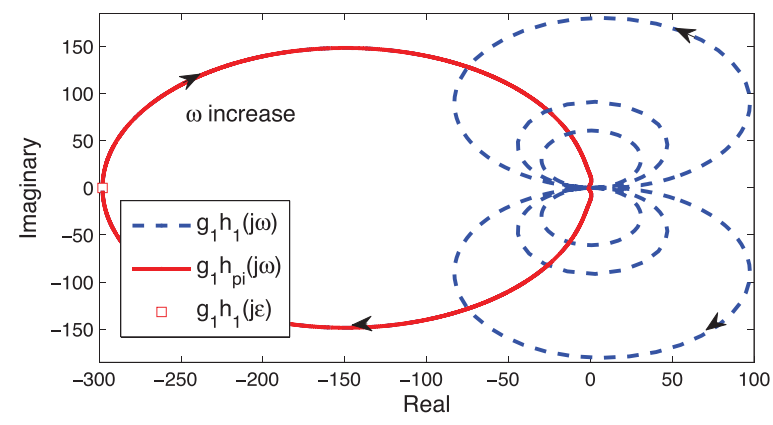

(a)

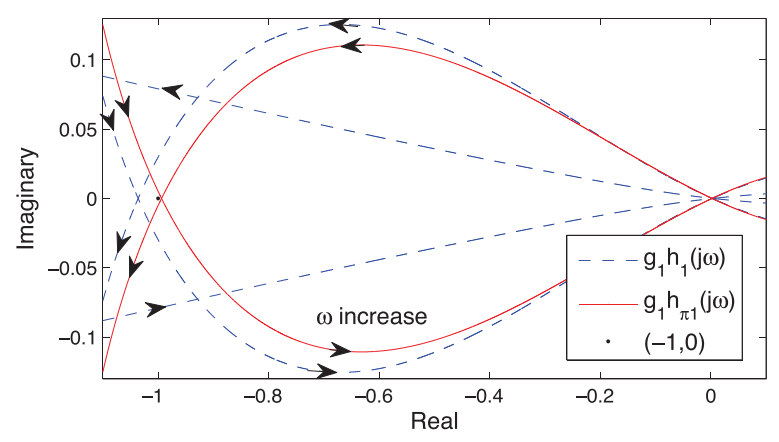

(b)

Fig. 7. Nyquist plot of $g_{1}(j \omega) h_{1}(j \omega)$ : red curve is the Nyquist plot of VSCHVDC system with one П-section DC cable model; blue curve is the Nyquist plot of the VSC-HVDC system with distributed parameter DC cable model; $\varepsilon=0.01[\mathrm{rad} / \mathrm{s}]$ (a) Full curve (b) Amplified around critical point $(-1,0)$.
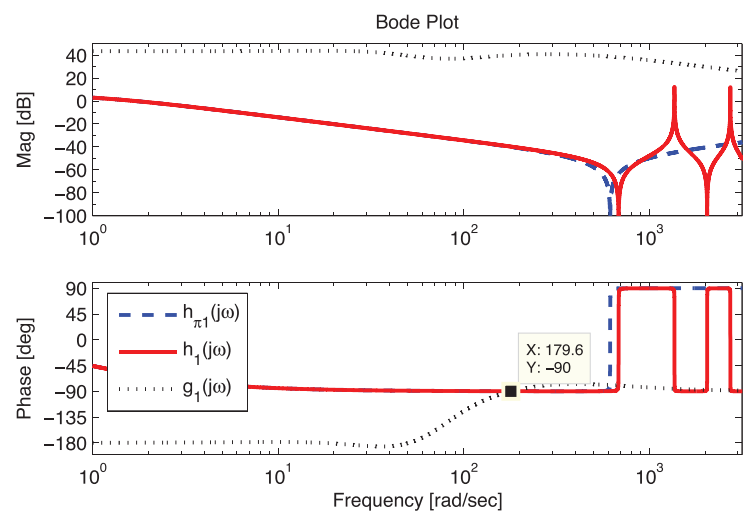

Fig. 8. Frequency characteristic curve of $g_{1}(j \omega), h_{1}(j \omega)$ and $h_{\pi 1}(j \omega)$.

implying that the VSC-HVDC system with distributed parameter cable model has a larger gain margin.

Fig. 8 shows that if the VSC-HVDC system with one $\Pi$-section is stable, then the phase crossover frequency must be lower than $\omega_{r f}$, where $h_{1}(j \omega)$ is similar to $h_{p i}(j \omega)$, and since $g_{1} h_{1}$ has a larger gain margin, the VSC-HVDC system with distributed parameter cable model is also stable. Consequently, the VSC-HVDC system with one $\Pi$-section DC cable model is stable, which is a sufficient condition for the VSC-HVDC system with distributed parameter cable model to be stable, but not vice versa.

For different operating points $P_{20}$ and for different SCRs, the open loop function $g_{1} h_{1}$ always has one unstable pole, which implies that the Nyquist curve should anti-clockwise encircle the critical point $(-1,0)$ once, so that the closed loop VSCHVDC system is stable. As shown in Fig. 7, the Nyquist curve should cross the negative real axis at the left of $(-1,0)$ in order to anti-clockwise encircle the critical point. Therefore, the larger magnitude of $\left|g_{1} h_{1}\left(j \omega_{p c}\right)\right|$ is, the better stability robustness the system will have. $\omega_{p c}$ is the phase cross-over frequency, i.e., the angle of $g_{1} h_{1}\left(j \omega_{p c}\right)$ is $-180[\mathrm{deg}]$.

Fig. 9 illustrates the difference in loop transfer function gain, for the two cases (distributed model and $\Pi$-section model) at $\omega_{p c}, \omega_{p c}^{\pi}$, respectively. Positive difference values imply that $\left|g_{1} h_{1}\left(j \omega_{p c}\right)\right|>\left|g_{1} h_{\pi 1}\left(j \omega_{p c}^{\pi}\right)\right|$. It shows that the VSC-HVDC system with one $\Pi$-section DC cable model always has worse stability robustness for different operating points $P_{20}$ and for different SCRs. Consequently, the VSC-HVDC system with a single $\Pi$-section DC cable is a safe choice in the evaluation of system stability under different $P_{20}$ and SCR values.

\section{B. Strong AC-Grid Environment}

For a VSC-HVDC system embedded in a strong AC-grid, the small signal stability analysis based on the Nyquist stability criterion is discussed in, e.g., [10]. It gives:

$$
\begin{aligned}
\hat{g}_{11} & =\frac{\alpha_{c} \omega_{n d} C_{1}\left(2 \zeta s+\omega_{n d}\right)}{C_{1} s^{2}\left(s+\alpha_{c}\right)+\alpha_{c} \omega_{n d} C_{1}\left(2 \zeta s+\omega_{n d}\right)+\frac{i_{D C 10}}{v_{D C 10}} s^{2}} \\
\hat{g}_{13} & =\frac{\frac{P_{10}}{E_{10} v_{D C 10}} s^{2}}{C_{1} s^{2}\left(s+\alpha_{c}\right)+\alpha_{c} \omega_{n d} C_{1}\left(2 \zeta s+\omega_{n d}\right)+\frac{i_{D C 10}}{v_{D C 10}} s^{2}} \\
\hat{g}_{15} & =-\frac{s^{2}}{C_{1} s^{2}\left(s+\alpha_{c}\right)+\alpha_{c} \omega_{n d} C_{1}\left(2 \zeta s+\omega_{n d}\right)+\frac{i_{D C 10}}{v_{D C 10}} s^{2}} \\
\hat{g}_{21} & =\frac{\alpha_{p}}{\left(C_{2} v_{D C 20} s+i_{D C 20}\right)\left(s+\alpha_{p}\right)} \\
\hat{g}_{23} & =\frac{\frac{P_{20}}{E_{20} s}}{\left(C_{2} v_{D C 20} s+i_{D C 20}\right)\left(s+\alpha_{p}\right)} \\
\hat{g}_{25} & =-\frac{v_{D C 20}}{C_{2} v_{D C 20} s+i_{D C 20}}
\end{aligned}
$$

The forward function $\hat{g}_{0}(s)=-1 /\left(\hat{g}_{15}+\hat{g}_{25}\right) \cdot \hat{G}(s)$, whose stability depends on the zero polynomial $Q_{3}(s)$ of $\hat{g}_{15}+\hat{g}_{25}$ :

$$
\begin{aligned}
Q_{3}(s)=\left(C_{1}+C_{2}\right) s^{3}+\left(C_{1} \alpha_{c}+\right. & \left.\frac{i_{D C 10}}{v_{D C 10}}+\frac{i_{D C 20}}{v_{D C 20}}\right) s^{2}+ \\
& +\alpha_{c} K_{p d} s+\alpha_{c} K_{i d} .
\end{aligned}
$$

To guarantee that the zeros of $Q_{3}(s)$ are located in the left half plane, the following inequality must hold:

$$
\frac{C_{1} \alpha_{c}+\frac{i_{D C 10}}{v_{D C 10}}+\frac{i_{D C 20}}{v_{D C 20}}}{C_{1}+C_{2}}>\frac{K_{i d}}{K_{p d}}=\frac{\omega_{n d}}{2 \zeta} .
$$

To minimize transmission losses, the DC voltage drop between two terminals should be kept small. Further, the bandwidth of the inner current loop $\alpha_{c}$ is designed to be ten times larger than the DC voltage loop. Therefore, the inequality (44) holds for all reasonable designs of PI-controllers, and the forward function $\hat{g}_{0}$ for all input-output combinations are thus stable in case of strong AC-grid environment.

In [10], two examples have been investigated, showing that in both cases with either the rectifier or the inverter working as DC voltage controller, the VSC-HVDC system is stable for three different cable distances: $\mathrm{d}=50 \mathrm{~km}, 150 \mathrm{~km}, 450 \mathrm{~km}$. The 


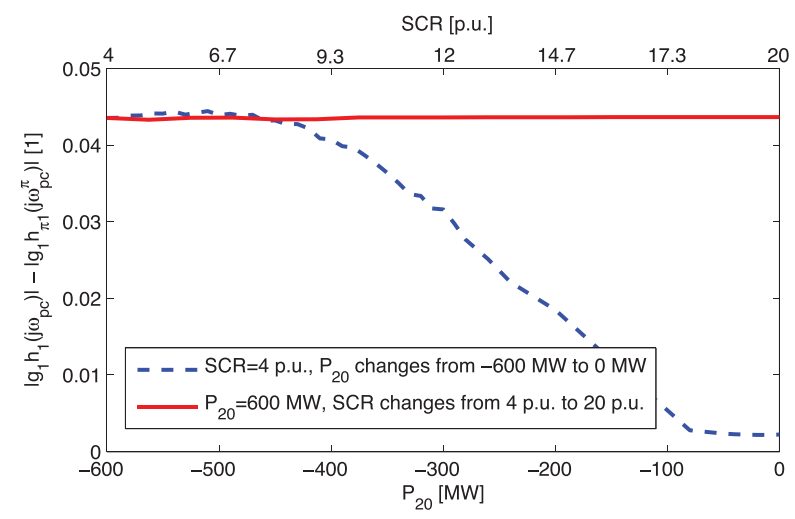

Fig. 9. Magnitude differences between $\left|g_{1} h_{1}\left(j \omega_{c}\right)\right|$ and $\left|g_{1} h_{p i}\left(j \omega_{c \pi}\right)\right|$, where the angle of both $g_{1} h_{1}\left(j \omega_{c}\right)$ and $g_{1} h_{p i}\left(j \omega_{c \pi}\right.$ are $-180^{\circ}$.

same result is obtained when the distributed parameter cable model is changed into one $\Pi$-section model.

\section{CONCLUSION}

A mathematical model for small-signal stability analysis of a two-terminal VSC-HVDC system embedded in a weak AC-environment has been presented. The system can be divided into two parts: the active power controlled VSC and the DC voltage controlled VSC with a DC cable model. The stability of the first part is analyzed by the small gain theorem, which shows that for SCR $>2.7$, with the parameter values from Table II, the active power controlled VSC embedded in a weak AC-environment will always be stable. The second part is analyzed by the Nyquist criterion. Due to the symmetric properties of the cable model, the block diagram of each input-output combination can be rewritten as in Fig. 2, where $g_{0}(s)$ is stable with reasonable design of the DC voltage PI-controller and SCR $>4$. The transfer function $g_{1}(s)$, is a rational function of ' $\mathrm{s}$ ' and the return path $h_{1}(s)$ is dissipative. A case study has been illustrated, showing that if the VSC-HVDC system with one $\Pi$-section cable model is stable, then the VSC-HVDC system with distributed parameter DC cable model is also stable, provided that $\left|h_{1}(j \omega)\right|>\left|h_{p i}(j \omega)\right|$ for $\omega<1 / d / \sqrt{l c / 2}$.

The proposed method proves that for long distance VSC-HVDC transmission or larger cable impedance density, the VSC-HVDC system with one $\Pi$-section cable model is sufficient for system stability evaluation. Also, the VSC-HVDC system with distributed parameter cable can provide accurate high-frequency response.

\section{APPENDIX A}

\section{State-Space Model of Active Power Controlled VSC}

The disturbance matrices $C_{w 1}$ and $D_{w 1}$ of the third order state-space model of active power controlled VSC are:

$$
\begin{aligned}
w & =C_{w 1} \cdot x_{a p}+D_{w 1} \cdot u_{a p} \\
C_{w 1} & =\frac{L_{g}}{k_{1}}\left[\begin{array}{cc}
1+a_{p l l} L_{g} \frac{P_{0}}{E_{0}^{2}} & -\left(a_{p}+a_{p l l}\right) L_{g} \frac{Q_{0}}{E_{0}^{2}} \\
a_{c} L_{g} K_{p a} & 1-a_{p} L_{g} \frac{P_{0}}{E_{0}^{2}}
\end{array}\right] .
\end{aligned}
$$

$$
\begin{aligned}
& \cdot\left[\begin{array}{ccc}
a_{p}-\frac{R_{g}}{L_{g}} & \omega_{0} & \omega_{0} \frac{P_{0}}{E_{0}^{2}}+\frac{Q_{0}}{E_{0}^{2}}\left(a_{p}+a_{p l l}-\frac{R_{g}}{L_{g}}\right) \\
-\omega_{0} & a_{c}-\frac{R_{g}}{L_{g}} & \left(a_{p l l}-\frac{R_{g}}{L_{g}}\right) \frac{P_{0}}{E_{0}^{2}}-\frac{Q_{0}}{E_{0}^{2}} \omega_{0}
\end{array}\right] \\
& D_{w 1}=\frac{1}{k_{1}}\left[\begin{array}{cc}
1+a_{p l l} L_{g} \frac{P_{0}}{E_{0}^{2}} & -\left(a_{p}+a_{p l l}\right) L_{g} \frac{Q_{0}}{E_{0}^{2}} \\
a_{c} L_{g} K_{p a} & 1-a_{p} L_{g} \frac{P_{0}}{E_{0}^{2}}
\end{array}\right] \text {. } \\
& \cdot\left[\begin{array}{cccc}
-\frac{a p}{E_{0}} L_{g} & 0 & 1 & 0 \\
0 & -a c K_{p a} L_{g} & 0 & 1
\end{array}\right] \\
& k_{1}=1+L_{g}\left(a_{p l l}-a_{p}\right) \frac{P_{0}}{E_{0}^{2}}- \\
& -L_{g}^{2}\left[a_{p} a_{p l l} \frac{P_{0}^{2}}{E_{0}^{4}}+a_{c}\left(a_{p}+a_{p l l}\right) K_{p a} \frac{Q_{0}}{E_{0}^{2}}\right] .
\end{aligned}
$$

The disturbance matrices $\tilde{C}_{w 1}$ and $\tilde{D}_{w 1}$ of the second order state-space model of active power controlled VSC are:

$$
\begin{aligned}
& w=\tilde{C}_{w 1} \cdot x_{a p n}+\tilde{D}_{w 1} \cdot u_{a p} \\
& \tilde{C}_{w 1}=\frac{L_{g}}{\tilde{k}_{1}}\left[\begin{array}{cc}
1+a_{p l l} L_{g} \frac{P_{0}}{E_{0}^{2}} & -\left(a_{p}+a_{p l l}\right) L_{g} \frac{Q_{0}}{E_{0}^{2}} \\
K_{p a} L_{g} \frac{R_{g}}{L_{g}} & 1+\left(\omega_{0} K_{p a}-a_{p} \frac{P_{0}}{E_{0}^{2}}\right) L_{g}
\end{array}\right] \text {. } \\
& \cdot\left[\begin{array}{cc}
a_{p}-\frac{R_{g}}{L_{g}} & \omega_{0} \frac{P_{0}}{E_{0}^{2}}+\frac{Q_{0}}{E_{0}^{2}}\left(a_{p}+a_{p l l}-\frac{R_{g}}{L_{g}}\right) \\
-\omega_{0} & \left(a_{p l l}-\frac{R_{g}}{L_{g}}\right) \frac{P_{0}}{E_{0}^{2}}-\frac{Q_{0}}{E_{0}^{2}} \omega_{0}
\end{array}\right] \\
& \tilde{D}_{w 1}=\frac{1}{\tilde{k}_{1}}\left[\begin{array}{cc}
1+a_{p l l} L_{g} \frac{P_{0}}{E_{0}^{2}} & -\left(a_{p}+a_{p l l}\right) L_{g} \frac{Q_{0}}{E_{0}^{2}} \\
K_{p a} L_{g} \frac{R_{g}}{L_{g}} & 1+\left(\omega_{0} K_{p a}-a_{p} \frac{P_{0}}{E_{0}^{2}}\right) L_{g}
\end{array}\right] \text {. } \\
& \cdot\left[\begin{array}{cccc}
-\frac{a p}{E_{0}} L_{g} & K_{p a} \omega_{0} L_{g} & 1 & 0 \\
0 & -\frac{R_{g}}{L_{g}} K_{p a} L_{g} & 0 & 1
\end{array}\right] \\
& \tilde{k}_{1}=1+L_{g}\left[\left(a_{p l l}-a_{p}\right) \frac{P_{0}}{E_{0}^{2}}+\omega_{0} K_{p a}\right]+ \\
& +L_{g}^{2}\left[a_{p l l}\left(\omega_{0} K_{p a}-a_{p} \frac{P_{0}}{E_{0}^{2}}\right) \frac{P_{0}}{E_{0}^{2}}+K_{p a} \frac{R_{g}}{L_{g}}\left(a_{p}+a_{p l l}\right) \frac{Q_{0}}{E_{0}^{2}}\right] .
\end{aligned}
$$

For a strong AC-grid, $L_{g} \rightarrow 0$ and thus both $C_{w 1}$ and $\tilde{C}_{w 1}$ are zero matrix and $D_{w 1}=\tilde{D}_{w 1}=\left[\begin{array}{cccc}0 & 0 & 1 & 0 \\ 0 & 0 & 0 & 1\end{array}\right]$. It means that for a strong AC-grid, $\Delta E_{d}=\Delta v_{s d}$ and $\Delta E_{q}=\Delta v_{s q}$.

\section{APPENDIX B}

\section{State-Space Model of VSC-HVDC System With ONE II-SECTION CABLE MODEL}

The state-space model of a VSC-HVDC system with one $\Pi$-section DC cable model is given below, where $\hat{C}=C+$ $d \cdot c / 2$ :

$$
\begin{aligned}
& \dot{x}_{d v}=A_{2} \cdot x_{d v}+B_{u 2} \cdot u_{d v}^{r e f}+B_{w 2} \cdot w \\
& w=C_{w 2} \cdot x_{d v}+D_{w 2} \cdot u_{d v} \\
& A_{2}=\left[\begin{array}{cccc}
-a_{c} & 0 & -2 \zeta \omega_{n d} \hat{C} \frac{v_{D C 0}}{E_{0}} a_{c} & \omega_{n d}^{2} \hat{C} \frac{v_{D C 0}}{E_{0}} a_{c} \\
0 & -a_{c} & 0 & 0 \\
\frac{E_{0}}{\hat{C} v_{D C 0}} & 0 & -\frac{i_{D C 0}}{\hat{C} v_{D c 0}} & 0 \\
0 & 0 & -1 & 0 \\
a_{f} E_{0} \frac{d \cdot c}{2} & 0 & a_{f} i_{D C 0} \frac{C}{\hat{C}} & 0 \\
0 & 0 & 0 & 0 \\
0 & 0 & \frac{1}{L_{D C}} & 0 \\
0 & 0 & 0 & 0
\end{array}\right.
\end{aligned}
$$




$$
\begin{aligned}
& \left.\begin{array}{cccc}
\frac{a_{c}}{E_{0}} & 0 & 0 & 0 \\
0 & 0 & 0 & 0 \\
0 & \frac{E_{0}}{\hat{C} v_{D C 0}} \frac{Q_{0}}{E_{0}^{2}} & -\frac{1}{\hat{C}} & 0 \\
0 & 0 & 0 & 0 \\
-a_{f} & a_{f} E_{0} \frac{d \cdot c}{\hat{C}} \frac{Q_{0}}{E_{0}^{2}} & a_{f} v_{D C 0} \frac{C}{\hat{C}} & 0 \\
0 & -a_{p l l} & 0 & 0 \\
0 & 0 & -\frac{R_{D C}}{L_{D C}} & -\frac{1}{L_{D C}} \\
0 & 0 & \frac{1}{\hat{C}} & \frac{i_{D D 0}}{\hat{C} v_{D C 0}^{a p}}
\end{array}\right] \\
& B_{u 2}^{T}=\left[\begin{array}{cccccccc}
2 \zeta \omega_{n d} \hat{C} \frac{v_{D C 0}}{E_{0}} a_{c} & 0 & 0 & 1 & 0 & 0 & 0 & 0 \\
0 & a_{c} K_{p a} & 0 & 0 & 0 & 0 & 0 & 0
\end{array}\right] \\
& B_{w 2}^{T}=\left[\begin{array}{cccc}
-a_{c} \frac{P_{0}}{E_{0}^{2}} & -a_{c} K_{p a} & \frac{E_{0}}{\hat{C} v_{D C 0}} \frac{P_{0}}{E_{0}^{2}} & 0 \\
0 & 0 & -\frac{E_{0}}{\hat{C} v_{D C 0}} \frac{Q_{0}}{E_{0}^{2}} & 0
\end{array}\right.
\end{aligned}
$$

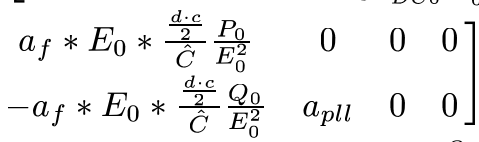

$$
\begin{aligned}
& C_{w 2}=\frac{L_{g}}{k_{2}}\left[\begin{array}{cc}
1+a_{p l l} L_{g} \frac{P_{0}}{E_{0}^{2}} & -a_{p l l} L_{g} \frac{Q_{0}}{E_{0}^{2}} \\
a_{c} L_{g} K_{p a} & 1-a_{c} L_{g} \frac{P_{0}}{E_{0}^{2}}
\end{array}\right] \text {. } \\
& \cdot\left[\begin{array}{cccc}
a_{c}-\frac{R_{g}}{L_{g}} & \omega_{0} & 2 \zeta \omega_{n d} \hat{C} \frac{v_{D C 0}}{E_{0}} a_{c} & -\omega_{n d}^{2} \hat{C} \frac{v_{D C 0}}{E_{0}} a_{c} \\
-\omega_{0} & a_{c}-\frac{R_{g}}{L_{g}} & 0 & 0
\end{array}\right. \\
& \left.\begin{array}{cccc}
-\frac{a_{c}}{E_{0}} & \omega_{0} \frac{P_{0}}{E_{0}^{2}}+\frac{Q_{0}}{E_{0}^{2}}\left(a_{p l l}-\frac{R_{g}}{L_{g}}\right) & 0 & 0 \\
0 & \left(a_{p l l}-\frac{R_{g}}{L_{g}}\right) \frac{P_{0}}{E_{0}^{2}}-\frac{Q_{0}}{E_{0}^{2}} \omega_{0} & 0 & 0
\end{array}\right] ; \\
& D_{w 2}=\frac{1}{k_{2}}\left[\begin{array}{cc}
1+a_{p l l} L_{g} \frac{P_{0}}{E_{0}^{2}} & -a_{p l l} L_{g} \frac{Q_{0}}{E_{0}^{2}} \\
a_{c} L_{g} K_{p a} & 1-a_{c} L_{g} \frac{P_{0}}{E_{0}^{2}}
\end{array}\right] \text {. } \\
& \cdot\left[\begin{array}{cccc}
-2 \zeta \omega_{n d} \hat{C} \frac{v_{D C 0}}{E_{0}} a_{c} L_{g} & 0 & 1 & 0 \\
0 & -a c K_{p a} L_{g} & 0 & 1
\end{array}\right] \\
& k_{2}=1+L_{g}\left(a_{p l l}-a_{c}\right) \frac{P_{0}}{E_{0}^{2}}+L_{g}^{2} a_{p l l} a_{c}\left(K_{p a} \frac{Q_{0}}{E_{0}^{2}}-\frac{P_{0}^{2}}{E_{0}^{4}}\right) .
\end{aligned}
$$

\section{REFERENCES}

[1] N. Flourentzou, V. Agelidis, and G. Demetriades, "VSC-based HVDC power transmission systems: An overview," IEEE Trans. Power Electron., vol. 24, no. 3, pp. 592-602, Mar. 2009.

[2] P. Bresesti, W. L. Kling, R. L. Hendriks, and R. Vailati, "HVDC connection of offshore wind farms to the transmission system," IEEE Trans. Energy Convers., vol. 22, no. 1, pp. 37-43, Mar. 2007.

[3] J. Svensson, Grid-Connected Voltage Source Converter-Control Principles and Wind Energy Applications. Gothenborg, Sweden: Chalmers University of Technology, 1998.
[4] S. Cole, J. Beerten, and R. Belmans, "Generalized dynamic VSC MTDC model for power system stability studies," IEEE Trans. Power Syst., vol. 25, no. 3, pp. 1655-1662, Aug. 2010.

[5] L. Zhang, "Modeling and control of VSC-HVDC links connected to weak ac system," Ph.D. dissertation, School Elect. Eng., Royal Inst. Technol., Stockholm, Sweden, 2010.

[6] G.-C. Hsieh and J. C. Hung, "Phase-locked loop techniques-a survey," IEEE Trans. Ind. Electron, vol. 43, no. 6, pp. 609-615, Dec. 1996.

[7] B. T. Ooi and X. Wang, "Voltage angle lock loop control of the boost type PWM converter for HVDC application," IEEE Trans. Power Electron., vol. 5, no. 2, pp. 229-235, Apr. 1990.

[8] L. Harnefors, M. Bongiorno, and S. Lundberg, "Input-admittance calculation and shaping for controlled voltage-source converters," IEEE Trans. Ind. Electron., vol. 54, no. 6, pp. 3323-3334, Dec. 2007.

[9] B. K. Bose, Power Electronics and Variable Frequency Drives. New York, USA: IEEE, 1997.

[10] Y. Song and C. Breitholtz, "Nyquist stability analysis of a VSC-HVDC system using a distributed parameter DC-cable model," presented at the 19th World Congr. Int. Fed. Autom. Control, Cape Town, South Africa, Aug. 2014.

[11] A. Jain, K. Joshi, A. Behal, and N. Mohan, "Voltage regulation with STATCOMs: Modeling, control and results," IEEE Trans. Power Del., vol. 21, no. 2, pp. 726-735, Apr. 2006.

[12] K. J. Astrom and R. M. Murray, Feedback System - an Introduction for Scientists and Engineers. Princeton, NJ, USA: Princeton University Press, 2008.

[13] H. K. Khalil, Nonlinear Systems, 3rd ed. Upper Saddle River, NJ, USA: Prentice-Hall, 2002.

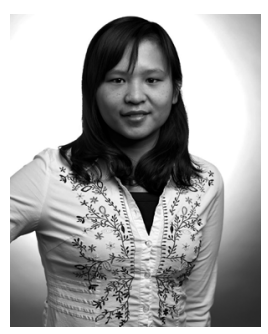

Yujiao Song received the M.Sc. degree in systems, control and mechatronics from Chalmers University of Technology, Gothenburg, Sweden, in 2011, where she is currently pursuing the Ph.D. degree in automatic control.

Her research interests include useful control methods as well as ways to analyze the stability for voltage-source-converter-based HVDC transmission systems.

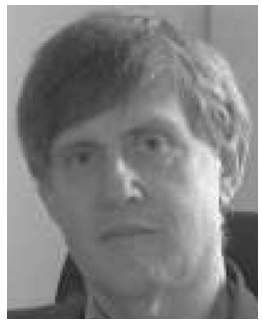

Claes Breitholtz received the Ph.D. degree in automatic control from Chalmers University of Technology, Gothenburg, Sweden, in 1982.

Currently, he is a Professor of Automatic Control in the Department of Signals and Systems, Chalmers University of Technology, after which he took up a position at ASEA (now ABB), where he was involved in the industrial application of adaptive control (within the NOVATUNE project) until 1985. After a short period as a Consulting Engineer, he returned to Chalmers in 1986 as an Associate Professor in automatic control, becoming a Professor in 2001. His research interests are mainly in the areas of model-based control of distributed parameter systems, model simplification methods, and stochastic optimal control. 